\title{
Assessment of sperm DNA in patients submitted the assisted reproduction technology procedures
}

\author{
Patrícia Miyuki Tsuribe ${ }^{1}$, João Ferreira Lima Neto², Marjorie de Assis Golim³ ${ }^{3}$ Camila de Paula Freitas Dell'Aqua², \\ João Paulo Issa ${ }^{1}$, Carlos Alberto Monte Gobbo ${ }^{1,4}$ \\ ${ }^{1}$ Clínica Endogin, Bauru/SP \\ 2Department of Animal Reproduction, Veterinary Medicine and Animal Science School, São Paulo State \\ University at Botucatu/SP \\ ${ }^{3}$ Blood Center, School of Medicine of the São Paulo State University at Botucatu/SP \\ ${ }^{4}$ Department of Urology, School of Medicine of the São Paulo State University at Botucatu/SP
}

\begin{abstract}
Objective: This study aimed to produce data on sperm quality while maintaining the integrity of sperm DNA samples taken from patients submitted to in vitro fertilization (IVF) procedures at our center, and determine whether increased levels of histones were associated with sperm DNA damage and decreased fertilization, cleavage, and pregnancy rates. Such findings might shed light on the physiology and outcomes of pregnancy.

Methods: Semen samples from 27 patients divided into two groups were analyzed. The case group included individuals offered IVF; the control group had subjects with normal spermograms. Sperm DNA structure was assessed through phosphorylated histone H2AX analysis by flow cytometry.

Results: The patients with altered sperm parameters had more histones in sperm chromatin than the individuals with normal sperm parameters.

Conclusion: Results indicated that increased levels of histone in sperm chromatin do not affect embryo production, but affect the cleavage rate, embryo quality, and might thus reduce pregnancy rates. The integrity of the paternal genome is of paramount importance in the initiation and maintenance of a viable pregnancy in patients treated with assisted reproduction technology procedures. Further studies on sperm diagnostic tests at a nuclear level might improve the treatment offered to infertile couples.
\end{abstract}

Keywords: Sperm DNA, Histones, Pregnancy, Fertilization rate, ICSI.

\section{INTRODUCTION}

The Sperm DNA integrity is essential for the accurate transmission of the genetic code, in addition to serving as an indicator of the quality of spermatogenesis and male fertility (Smith et al., 2007). Disturbances in the integrity of chromatin are characterized by the presence of breakages in single or double strands of sperm DNA, which lead to the formation of denatured segments (Rybar et al., 2004).

Increased susceptibility to denaturation is suggestive of chromatin structure heterogeneity and has been linked to disturbances in spermatogenesis, abnormal morphology (Enciso et al., 2011), decreased sperm concentration and motility (Benchaib et al., 2003), hampered embryonic development, and decreased fertility (Hallap, 2005). Although many authors have reported high DNA fragmentation levels in infertile patients with abnormal spermatozoa parameters (Aitken \& De luliis, 2007; Henkel, 2007), some patients with normal semen parameters and idiopathic infertility have been shown to present higher levels of damaged sperm DNA (Saleh et al., 2003; Cohan et al., 2006). More than $30 \%$ of these patients require longer times to establish a normal pregnancy, more assisted reproduction cycles, and are at a higher risk of experiencing a miscarriage (Giwercman et al., 2010). Sperm DNA damage can occur within the testes, the epididymis or in ejaculates. Its multifactorial etiology may include extrinsic and intrinsic factors (Zini \& Sigman, 2009; Sakkas \& Alvarez, 2010). Different hypotheses have been proposed to explain the origin of DNA damage in mature sperm of infertile men, including abnormalities in chromatin condensation, microdeletions, aneuploidies, chromosome rearrangements, breakages in single or double strand DNA, apoptosis, and oxidative stress (Gorczca et al., 1993; Manicardi et al., 1995; Sakkas et al., 1999; Barroso et al., 2000; Cho et al., 2001; Souza et al., 2010). In addition to biological factors such as infection, sexually transmitted diseases, obesity, diabetes, age, lifestyle factors including smoking (Pacey, 2010) may also affect chromatin integrity.

Sperm chromatin is organized within a highly condensed and compressed structure that significantly protects the DNA against damage while making it promptly available to the ooplasm (Bjorndahl \& Kvist, 2010). Sperm chromatin undergoes a series of changes during spermatogenesis, with the first important event being the hyperacetylation of histones to facilitate the disassembly of the nucleosome. During this process, sperm DNA is extensively complexed with transition proteins. At the end of spermiogenesis these transition proteins are replaced by protamines, leaving the DNA in human sperm chromatin a fraction in nucleohistones and a fraction in nucleoprotamines, with $15 \%$ of the DNA bound to histones and $85 \%$ bound to protamines (Bjorndahl \& Kvist, 2010; Oliva, 2006). Infertile men have increased histone to protamine ratios when compared to fertile controls. This change in the ratio, which consists of abnormal chromatin packaging, increases the susceptibility of sperm DNA to external stress, due to the lower compaction of chromatin (Zini \& Sigman, 2009; Ozmen et al., 2007).

In recent years, rapid advances in molecular biology have led to the development of numerous tests to assess various aspects of DNA and chromatin quality. The tests are divided into direct and indirect assays. Direct assays include TUNEL, the neutral comet assay, and in situ nick translation; indirect assays include the alkaline comet assay, the sperm chromatin structure assay (SCSA), and the sperm chromatin dispersion (SCD) test (Lewis et al., 2008). These methods might detect cases of infertility missed in semen analysis. Studies have shown that levels of DNA damage in mature spermatozoa are significantly correlated with sperm fertilization potential. DNA damage analysis may help predict the success of pregnancy and birth produced by natural and assisted reproduction technology (ART) procedures. Moreover, characterizing normal levels of DNA damage in normal sperm might help prevent the use of samples with high levels of DNA damage in ART procedures (Tesarik et al., 2004). 
This study aimed to produce data on sperm quality while maintaining the integrity of sperm DNA samples taken from patients submitted to in vitro fertilization (IVF) procedures at our center, and determine whether increased levels of histones were associated with sperm DNA damage and decreased fertilization, cleavage, and pregnancy rates. Such findings might shed light on the physiology and outcomes of pregnancy.

\section{MATERIALS AND METHODS}

Twenty-one couples were offered intracytoplasmic sperm injections (ICSI) at the Endogin Clinic in Bauru, Brazil. Individuals who smoked, drank alcohol, or had an infertile partner were excluded. Ejaculated semen samples from six individuals with normal findings on physical examination - normal volume, absence of varicocele, and history of pregnancy with a partner without using ART procedures within the last 12 months - were used as controls. Only the samples and data of the individuals who gave consent to participating in the study were used.

The patients referred to ICSI underwent controlled ovarian stimulation along with suppression of the hypothalamic-pituitary axis. Pituitary suppression was carried out between days 18 and 22 of the menstrual cycle with a gonadotropin-releasing hormone analogue (Lupron TM Kit, Abbott SA, Paris, France). Fourteen to twenty days later, the patients were started on ovarian stimulation with follicle-stimulating hormone (TM Gonal-F, Serono, Geneva, Switzerland) at dosages ranging between 150 and 300 IU depending on the patient's age. Induction was followed with serial transvaginal ultrasound scans. When the dominant follicle reached $18 \mathrm{~mm}$ or at least two follicles reached 16 to $18 \mathrm{~mm}$ in diameter, the patients were administered 250 ug of recombinant human chorionic gonadotropin (rhCG) (Ovidrel, Serono, Geneva, Switzerland) to enable final follicular maturation. Follicular aspiration was performed approximately 34 to 36 hours after the administration of rhCG. The recovered oocytes were denuded and incubated in the initial culture medium (G1Plus, Vitrolife, Gothenburg, Sweden), covered with paraffin oil (Ovoil, Vitrolife) previously equilibrated at $\mathrm{pH} 7.2-7.4$ and kept at $37^{\circ} \mathrm{C}$.

Oocyte morphology was assessed and patients with most of their oocytes showing a perivitelline space, extrusion of the first polar body (PB) MII, and an intact oolemma were selected, administered microinjections, and included in the case group.

Semen samples were obtained by masturbation after a period of three to five days of abstinence from the day of oocyte aspiration. The collected material was kept for 30 minutes at room temperature and after liquefaction the samples were macroscopically assessed for color, volume, viscosity, and liquefaction. Then microscopic analysis was performed to determine sperm concentration and motility. Normal values were defined as a concentration $>15$ million/ml or $39 \times 106 /$ ejaculate and motility $>32 \%$ (progressive motility) and $>40 \%$ (total motility). Morphology was considered normal when $>4 \%$ according to the Tygerberg criteria.

Portions of the semen samples were centrifuged at 300 $\mathrm{g}$ for seven minutes and were then frozen. Seminal plasma was discarded and the seminal pellet was washed twice in PBS at a pH of 7.4, resuspended at a final concentration of $5 \times 106 / \mathrm{ml}$, and stored at $-1960 \mathrm{C}$ for sperm chromatin analysis. The same procedure was performed with the semen samples of the control group.

Intracytoplasmic sperm injection (ICSI) was performed according to the standard technique described by Palermo et al., (1992). Embryo quality was assessed based on the modified morphological criteria described by Veeck (1991), including items such as number and symmetry of blastomeres and degree of fragmentation. Samples assigned to Grade I had no fragmentation and equal blastomeres; samples on Grade II had $<20 \%$ fragmentation and equal blastomeres; samples on Grade III had $<20 \%$ fragmentation and unequal blastomeres; samples on Grade IV had $>20 \%$ fragmentation and unequal blastomeres; and samples on Grade $V$ had > 50\% fragmentation.

Three days after oocyte aspiration, the embryos were selected for transfer. Fourteen days after embryo transfer the patients were tested for beta hCG levels to verify whether embryo implantation was successful. Ultrasound scans were performed in the 6th week of pregnancy to confirm fetus viability. Clinical pregnancy was defined when a gestational sac was detected in ultrasound scans. Clinically pregnant individuals who lost their fetuses before the 12 th week of pregnancy were categorized as cases of miscarriage.

Tests to determine the presence of histones in sperm chromatin

A fraction of the semen samples was tested with the Apoptosis, DNA Damage and Cell Proliferation Kit (BD Biosciences, catalog number 562253). Analysis by flow cytometry was performed according to the manufacturer's instructions. Sperm samples were thawed and diluted in $1 \mathrm{ml}$ buffer ( $1 \times 106 \mathrm{sperm})$ and centrifuged at $250 \mathrm{~g}$ for five minutes. The supernatant was discarded and the pellet resuspended in 100 ul BD Cytofix for fastening and Cytosperm (permeabilizing solution) and kept at room temperature for 30 minutes. Then it was washed with $1 \mathrm{ml} 1 \times$ BD Perm / Wash Buffer, and the supernatant was again removed and the pellet resuspended in 20ul BDPerm / Wash Buffer plus antibody Alexa Fluor 647Mouse anti-H2AX (pS139.5 $\mathrm{ll} /$ test). The samples were incubated for 20 minutes at room temperature. After 60 minutes, the samples were washed and resuspended in $1 \mathrm{ml}$ of $1 \times$ BD Perm / Wash Buffer and evaluated in the flow cytometer (FACScalibur, Becton Dickinson, San Jose, California, USA). A minimum of 100,000 cells per sample were assessed.

\section{Statistical Analysis}

Statistical analysis was performed using software package GraphPad Prism Version 5.00 for Windows (GraphPad Software, San Diego California USA, www.graphpad.com). The Kolmogorov-Smirnov test was used to verify the normality and homogeneity of variance when the samples had parametric data. The t-test was used the samples had non-parametric data. The Mann-Whitney test or the Kruskal-Wallis test were performed followed by Dunn's test. Significance was attributed to events with a $P<0.05$. Fisher's exact test was used to assess embryo quality and percentage of histones in relation to embryo quality.

\section{RESULTS}

The semen samples of 27 subjects were analyzed and divided into two groups separating patients offered IVF and healthy controls with normal spermograms. No statistically significant differences were found (Figure $1 \mathrm{~A}$ ) between case and control group subjects. A trend was observed $(P=0.0808)$ in which IVF patients $(n=21 ; 3.9 \pm 1.4)$ had a higher incidence of histones in sperm DNA chromatin than controls ( $n=6 ; 0: 48 \pm 0: 11)$. The impact of the level of histones in male fertility was further analyzed in terms of positive and negative cases of clinical pregnancy (Figure 1B). Again, no statistically significant difference was found in clinical pregnancy rates $(P=0.2486)$. This may have occurred because one patient with high levels of histones was positive for clinical pregnancy. Patients were also assessed for semen quality before freezing (Figure 1C), but no sta- 
patient $x$ control

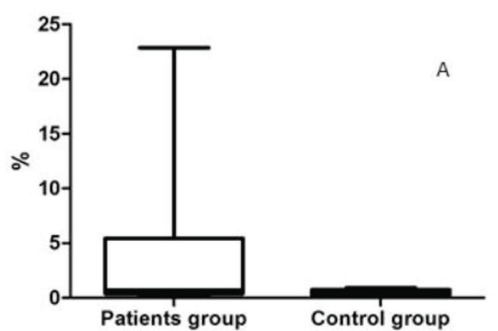

fertility

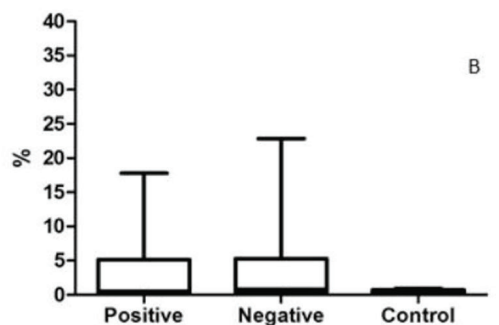

semen quality

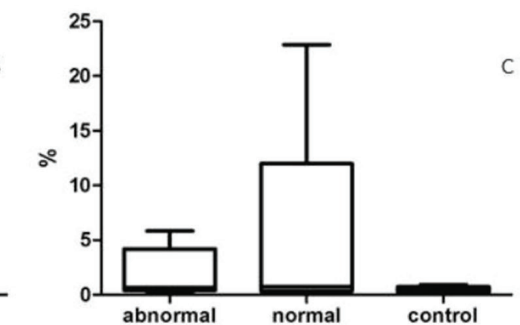

Figure 1: Representation of Whisker plots (min to max) of the data obtained from the analysis of histones in the sperm chromatin of patients undergoing in vitro fertilization procedures versus controls - males whose partners have had spontaneous pregnancies within less than 12 months (A). Patients positive for pregnancy versus individuals negative for pregnancy versus controls (B); Patients grouped according to sperm quality into abnormal, normal, and controls (C).

cleavage rate

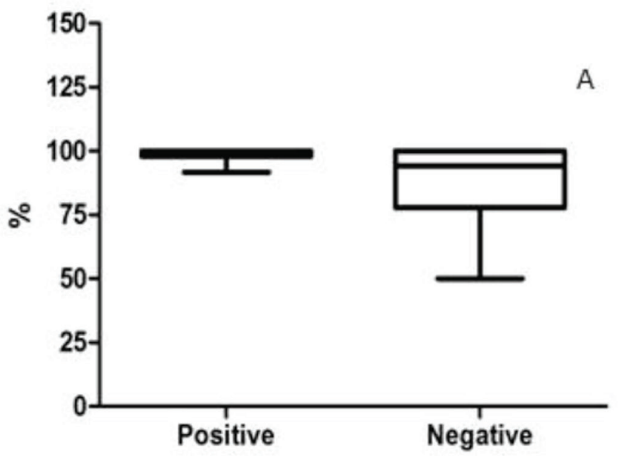

rate transferable embryos

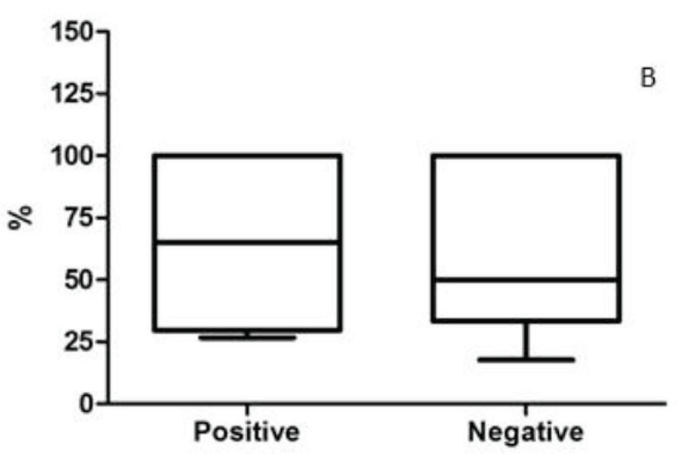

Figure 2: Representation of Whisker plots (min to $\max$ ) of the data obtained from the analysis of histones in the sperm chromatin of patients undergoing in vitro fertilization procedures grouped into subjects positive for pregnancy versus individuals negative for pregnancy in relation to cleavage rates (Fig A); and rate of transferable embryos (Fig B).

\section{Embryo quality}

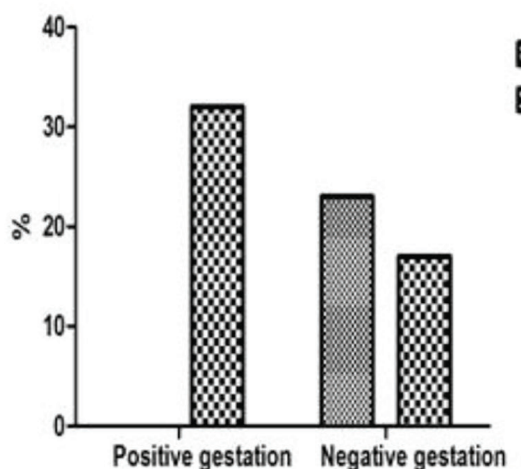

$\%$ of histones in the embryo quality

Low quality embryos $\mathbf{W}$ High quality embryos

A

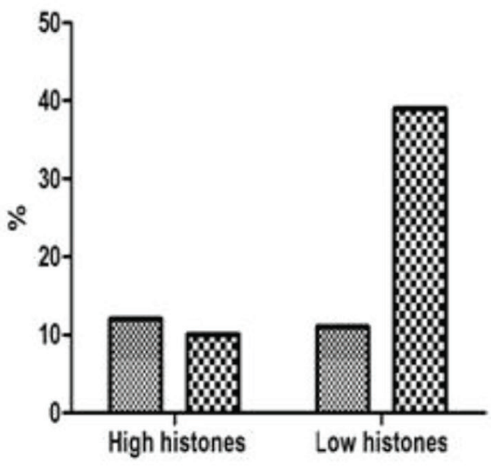

Low quality embryos

$\mathbf{x}$ High quality embryos

\section{B}

Figure 3: Graphs representing the analysis of embryo quality versus pregnancy using Fisher's exact test (fig A). Thirty-two embryos of Grades I and II (good quality) resulted in pregnancy, while 40 embryos - 17 in Grades I and II and 23 in Grades III and IV (poor quality) - did not result in pregnancy. Concerning the level of histones in sperm chromatin, 22 transferred embryos (10 of good and 12 of poor quality) had high histone levels ( $>1 \%$ ) and 50 (39 good and 11 poor quality embryos) had low histone levels (<0.99\%).

tistically significant differences were found $(P=0.3757)$. However, patients considered to have normal quality semen $(n=13)$ had higher histone levels (5.4 \pm 2.2$)$ than controls ( $n=6 ; 00: 48 \pm 00: 11$ ), while individuals with altered semen quality had only a slight increase in histone levels $(n=8 ; 1.8 \pm 0.8)$.

Cleavage rate $P=0.0506$ (Figure 2A) and the percentage of histones in sperm chromatin influenced the results. Patients with positive pregnancies $(98.61 \pm 1.38)$ had higher cleavage rates than individuals negative for pregnancy $(87.98 \pm 4.0)$. No statistically significance difference
$(P=0.1245)$ was observed in terms of the percentage of transferable embryos $(64.57 \pm 13.6$ versus $57.6 \pm 8.0$, for individuals positive and negative for pregnancy, respectively), as shown in Figure 2B.

Embryo quality was also assessed. As expected, clinical pregnancy rates were higher among individuals with higher quality embryos (Grades I and II) than in subjects with poorer quality embryos (Grades III and IV), as shown in Figure 3A $(P<0.001)$. The presence of histones in sperm chromatin significantly interfered with the quality of the transferred embryos (Figure 3B). Better quality embryos 
were produced from patients with lower sperm chromatin histone levels $(P=0.0123)$.

\section{DISCUSSION}

Oocytes fertilized by sperm with DNA alterations may significantly impair embryonic development and fetal health (Evenson et al., 2002), in addition to transmitting mutations that might affect the fertility of the next generation (Aitken \& Krausz, 2001). Thus, chromatin integrity plays a vital role in the paternal contribution to the production of a healthy fetus.

This study compiled data on chromatin alterations that may compromise the viability of sperm used in IVF. These changes are basically caused by apoptosis during spermatogenesis, breaks during chromatin remodeling, and fragmentation induced by reactive oxygen species (Tavalee et al., 2009; Sakkas \& Alvarez, 2010).

In recent years, the evaluation of sperm DNA fragmentation was recognized as a predictor of the likelihood of conception. The capacity for natural conception decreases in sperm with abnormal chromatin (Spano et al., 2000; Sergerie et al., 2005). Henkell et al. (2004) and Simon et al. (2011) found that patients treated repeatedly with conventional IVF experienced negative effects on fertilization and blastocyst development in the course of pregnancy. Reports on ICSI have published contradictory results. Some groups found (statistically significant) negative associations between the percentage of sperm with fragmented DNA and fertilization rates with ICSI (Lopes et al., 1998; Simon et al., 2013), while other authors did not observe differences in the fertilization rate but reported negative effects upon pregnancy rates (Benchaib et al., 2003) or embryo quality (Muriel et al., 2006). Higher levels of DNA fragmentation have been described as a cause for low quality embryo implantation and miscarriage (Tesarik et al., 2004; Evenson et al., 2002; Borini et al., 2006). Conversely, some studies did not describe sperm DNA fragmentation as having a role in ICSI results (Bungum et al., 2004; Nicopoullos et al., 2008). These authors looked into DNA fragmentation in the total population of sperm (morphologically abnormal and normal), but during the ICSI procedure, only sperm with good motility and morphology was selected for injection. The inadvertent selection of sperm cells based solely on normal motility and morphology without considering DNA integrity may yield undesired outcomes in ICSI procedures, which might impair not only embryonic development, but also the resulting offspring. Greco et al. (2005) showed that microinjection of sperm with fragmentation above $15 \%$ as analyzed using TUNEL resulted in a pregnancy rate of $5.6 \%$ versus $44.4 \%$ when sperm DNA fragmentation was below $6 \%$.

The results analyzed in this study showed that even men with normal sperm counts may have high levels of alteration in the compaction of chromatin, which means it might take longer to have a successful pregnancy, thus increasing the chances of having more ART cycles and the risk of having a miscarriage. Tests showed that these alterations were not related to fertilization rates, but to significant reductions in embryo quality and consequently in the pregnancy rates attained with ICSI cycles.

Although contradictory, no associations were found between embryo quality and sperm DNA damage measured by sperm chromatin structure assay (SCSA) and TUNEL (Irvine et al., 2000; Larson et al., 2000; Benchaib et al. 2003; Huang et al., 2005; Benchaib et al., 2007; Frydman et al., 2008), despite the different test sensitivities and the types of DNA damage measured specifically in each test.

There is currently a threshold assigned to percent DNA fragmentation in a sample above which pregnancy becomes unlikely. This threshold varies between $12 \%$
(Duran et al., 2002) and 36.5\% (Henkel et al., 2003), and has been described as $15 \%$ (Benchaib et al., 2007) and 20\% (Sergerie et al., 2005). Many are the possible reasons for the discrepant thresholds: different study populations (intrauterine insemination (IUI), in vitro fertilization (IVF) and/or intracytoplasmic sperm microinjection (ICSI); type of sperm used (obtained after washing or selected); or the type of count (operator or by flow cytometry).

Despite these observations, sperm with damaged DNA can preserve the potential for fertilization and produce viable embryos (Tesarik et al., 2004). While the oocyte might repair the damaged paternal DNA, this ability is limited and depends on the level of DNA damage (Fatehi et al., 2006). DNA damage may predispose the developing embryo to mutations and induce disease in the offspring (Aitken \& Krausz, 2001). Sperm DNA integrity is an important factor in the overall success of ART procedures. Although several studies have demonstrated the importance of assessing chromatin integrity and thresholds have been set to assess fertility levels, this parameter has not been evaluated routinely in andrology laboratories. This is possibly due to the complex protocols and expensive equipment - the likes of fluorescence microscopes and flow cytometers - required to run such tests.

Successful ART procedures rely on many factors other than semen quality and the ability of sperm to merge with the oolemma, such as the oocyte, uterine receptivity, and maternal immune status.

\section{CONCLUSIONS}

Changes in chromatin structure negatively affect the clinical outcomes of ICSI procedures. Clinical evidence suggests that these changes may curtail the effect of ART procedures even in men with normal sperm. The impact of chromatin structure alterations on fertilization rates is still controversial, but there is agreement over the negative effects it produces on embryonic development and pregnancy rates.

The predictive value of a DNA fragmentation test consists of a sum of factors, such as: 1 ) whether fragmentation occurred in single or double-stranded DNA (single offers better prognosis); 2) percentage of sperm with damaged DNA; higher percentages mean the likelihood of the egg being fertilized by sperm with intact DNA is reduced; 3 ) degree of DNA fragmentation in sperm (more intense fragmentation means DNA is less likely to be repaired); 4) primary or secondary damage; 5) type of test used (direct or indirect); 6) whether the damage affects encoding sequences (relatively low likelihood, as more than $90 \%$ of the DNA consists of non-encoding sequences); 7) the ability of the oocyte to repair the damage; 8 ) ability of the embryo to repair the damage; 9) number of oocytes in metaphase II; and 10) sample processing. It is also important to remember that although the 2006 Practice Committee Guidelines of the American Society for Reproductive Medicine (ASRM) accepted that sperm DNA fragmentation is more common in infertile men and that it may contribute to poor reproductive performance, there is no proven role for routine testing of DNA integrity in the evaluation of infertility, as current findings are enough only to suggest, rather than confirm, an association between sperm DNA testing and ART procedure outcomes.

The lack of an indication for routine testing was supported in a review published by Collins et al. (2008). More studies are needed to verify the association of DNA damage test results and the clinical outcomes of ART procedures. Oocyte quality may also be a major determinant on the negative effects introduced by sperm DNA damage. 


\section{CONFLICT OF INTERESTS}

No conflict of interest have been declared.

\section{Corresponding author:}

Patrícia Miyuki Tsuribe

Clínica Endogin, Bauru/SP - Brazil

E-mail:pmtsuribe@ig.com.br

\section{REFERENCES}

Aitken RJ, De luliis GN. Origins and consequences of DNA damage in male germ cells. Reprod BioMed Online 2007; 14: 727-33.

Aitken RJ, Krausz C. Oxidative stress, DNA damage and the Y chromosome. Reproduction 2001; 122: 497-506.

Barroso G, Morshedi M, Oehninger S. Analysis of DNA fragmentation, plasma membrane translocation of phosphatidylserine and oxidative stress in human spermatozoa. Hum Reprod 2000; 1338-44.

Benchaib M, Braun V, Lornage J, Hadj S, Salle B, Lejeune $H$, Guérin JF. Sperm DNA fragmentation decreases the pregnancy rate in an assisted reproductive technique. Hum Reprod 2003; 18: 1023-8.

Benchaib M, Lornage J, Mazoyer C, Lejeune $H$, Salle B, François Guerin J. Sperm deoxyribonucleic acid fragmentation as a prognostic indicator of assisted reproductive technology outcome. Fertil Steril 2007; 87: 93-100.

Bjorndahl L, Kvist U. Human sperm chromatin stabilization: a proposed model including zinc bridges. Mol Hum Reprod. 2010; 23-9.

Borini A, Tarozzi N, Bizzaro D, Bonu MA, Fava L, Flamingni C, Coticchio G. Sperm DNA fragmentation: paternal effect on early post-implantation embryo development in ART. Hum Reprod. 2006; 21, 2876-81.

Bungum M, Humaidan P, Spano M, Jepson K, Bungum L, Giwerchman A. The predictive value of sperm chromatin structure assay (SCSA) parameters for the outcome of intrauterine insemination, IVF and ICSI. Hum Reprod. 2004; 19: $1401-8$.

Cho C, Willis WD, Goulding EH, Jung-Ha, Choi YC, Hecht NB, Eddy EM. Haploinsufficiency of protamine-1 or -2 causes infertility in mice. Nat Genet 2001; 28: 82-6. Cohan KR, Griffin JT, Lafromboise M, De Jonge CJ, Carrell DT. Comparison of cromatin assays for DNA fragmentation evaluation in human sperm. J Androl 2006; 27:53-9.

Collins JA, Barnhart KT, Schlegel PN. Do sperm DNA integrity tests predict pregnancy with in vitro fertilization? Fertil Steril. 2008; 823-31.

Duran EH, Morshedi M, Taylor S, Oehninger S. Sperm DNA quality predicts intrauterine insemination outcome: a prospective cohort study. Hum Reprod 2002; 17: 3122-8.

Enciso M, Iglesias M, Galán I, Sarasa J, Gosálvez A, Gosálvez $\mathrm{J}$. The ability of sperm selection techniques to remove single- or double -strand DNA damage. Asian J Androl 2011; 13: 764-8.

Evenson DP, Larson KL, Jost LK. Sperm chromatin structure assay: its clinical use for detecting sperm DNA fragmentation in male infertility and comparisons with other techniques. J Androl 2002; 23: 25-43.
Fatehi AN, Bevers MM, Schoevers E, Roelen BA, Colenbrander B, Gadella BM. DNA damage in bovine sperm does not block fertilization and early embryonic development but induces apoptosis after the first cleavages. J Androl 2006; 27: 176-88.

Frydman N, Prisant N, Hesters L, Frydman R, Tachdjian G, Cohen-Bacrie P, Fanchin R. Adequate ovarian follicular status does not prevent the decrease in pregnancy rates associated with high sperm DNA fragmantation. Fertil Steril 2008; 89: 92-7.

Giwercman A, Lindstedt L, Larsson M, Bungum M, Spano M, Levine RJ, Rylander L. Sperm chromatin structure assay as an independent predictor of fertility in vivo: a case-control study. Int J Androl 2010; 33:221-7.

Gorczca W, Traganos F, Jesionowska H, Darzynkiewicz Z. Presence of DNA strand breaks and increased sensitivity of DNA in situ to denaturation in abnormal human sperm cells: analogy to apoptosis of somatic cells. Exp Cell Res. 1993; 207: 202-5.

Greco E, Scarcelli F, Iacobelli M, Rienzi L, Ubaldi F, Ferrero S, Franco G, Anniballo N, Mendoza C, Tesarik J. Efficiente treatment of infertility due to sperm DNA damage by ICSI with testicular spermatozoa. Hum Reprod. 2005; 20: 22630.

Hallap T. Assessment of sperm attributes of frozen-thawed AI doses from Swedish and Estonian dairy bulls sires. Doctoral thesis (Divison of comparative Reproduction, Obstetrics and Udder Health) - Department of Clinical Sciences - Swedish University of Agricultural Sciences. Uppsala. 2005.

Henkel R. DNA fragmentation and its influence on fertilization and pregnancy outcome. In: Oehninger SC, Kruger TF, eds. Male infertility: diagnosis and treatment. London, UK: Informa Healthcare; 2007. p. 49-72.

Henkel R, Hajimohammad M, Stall T, Hoogendijk C, Mehnert $C$, Menkveld $R$, Gips $H$, Schill WB, Kruger TF. Influence of deoxyribonucleic acid damage on fertilization and pregnancy. Fert Steril. 2004; 81: 965-72.

Henkel R, Kierspel E, Hajimohammad M, Stalf T, Hoogendijk C, Mehnert C, Menkveld R, Schill WB, Kruger TF. DNA fragmentation of spermatozoa and assisted reproduction technology. Reprod Biomed Online. 2003; 7: 477-84.

Huang $X$, Halicta HD, Traganos F, Tanaka T, Kurose A, Darzynkiewicz Z. Cytometric assessment of DNA damage in relation to cell cycle phase and apoptosis. Cell Prolif 2005 ; 38: 223-43.

Irvine DS, Twigg JP, Gordon EL, Fulton N, Milne PA, Aitken RJ. DNA integrity in human spermatozoa: relationships with semen quality. J Androl. 2000; 21: 33-44.

Larson KL, DeJonge CJ, Barnes AM, Jost LK, Evenson DP. Sperm chromatin structure assay parameters as predictors of failed pregnancy following assisted reproductive techniques. Hum Reprod. 2000; 15: 1717-22.

Lewis SE, Agbaje I, Alvarez J. Sperm DNA tests as useful adjuncts to semen analysis. Syst Biol Reprod Med. 2008; 54:111-25.

Lopes S, Jurisicova A, Casper RF. Gamete-specific DNA 
fragmentation in unfertilized human oocytes after intracytoplasmatic sperm injection. Hum Reprod. 1998; 13: 703-8.

Manicardi GC, Bianchi PG, Pantano S, Azzoni P, Bizzaro D, Bianchi U, Sakkas D. Presence of endogenous nicks in DNA of ejaculated human spermatozoa and its relationship to chromomycin A3 accessibility. Biol Reprod. 1995; 52: 864-7.

Muriel L, Garrido N, Fernandez JL, Remohi J, Pellicer A, de Los Santos MJ, Meseguer M. Value of the sperm deoxyribonucleic acid fragmentation level, as measured by the sperm chromatin dispersion test, in the outcome of in vitro fertilization and intracytoplasmatic sperm injection. Fertil Steril. 2006; 85: 371-83.

Nicopoullos JD, Gilling-Smith C, Almeida PA, Homa S, Norman-Taylor JQ, Ramsay JW. Sperm DNA fragmentation in subfertile men: the effect on the outcome of intracytoplasmatic sperm injection and correlation with sperm variables. BJU Int 2008; 101:1553-60.

Oliva R. Protamines and male infertility. Hum Reprod Update. 2006; 417-35.

Ozmen B, Koutjaki N, Youssry M, Diedrich K, Al-Hasani S. DNA damage of human spermatozoa in assisted reproduction: origins, diagnosis, impacts and safety. Reprod Biomed Online. 2007; 14: 384-95.

Pacey AA. Environmental and lifestyle factors associated with sperm DNA damage. Hum Fertil (Camb). 2010; 13: 189-93.

Palermo G, Joris H, Devroey P, Van Steirteghem AC. Pregnancies after intracytoplasmic injection of single spermatozoon into an oocyte. Lancet. 1992; 340:17-8.

Rybar R, Faldicova L, Faldyna M, Machatkova M, Rubes J. Bull and boar sperm DNA integrity evaluated by sperm chromatin structure assay in the Czech Republic. Vet. Med. 2004; 49: 1-8.

Sakkas D, Alvarez, JG. Sperm DNA fragmentation mechanisms of origin, impact on reproductive outcome, and analysis. Fertil Steril; 2010:93: 1027-36.

Sakkas D, Mariethoz E, Manicardi GC, Bizzaro D, Bianchi PG, Bianchi U. Origin of DNA damage in ejaculat- ed human spermatozoa. Rev Reprod. 1999; 4: 31-7. Saleh RA, Agarwal A, Sharma RK, Said TM, Sikka SC, Thomas AJ. Evaluation of nuclear DNA damage in spermatozoa from infertile men with varicocele. Fertil Steril. 2003; 80: $1431-6$.

Sergerie M, Laforest G, Bujan L, Bissonnette F, Bleau G. Sperm DNA fragmentation: threshold value in male fertility. Hum Reprod. 2005; 20: 3446-51.

Simon L, Castilho J, Oliva R, Lewis SE. Relationships between human sperm protamines, DNA damage and assisted reproduction outcomes. Reprod Biomed Online 2011; 23:724-34.

Simon L, Proutski I, Stevenson M, Jennings D, McManus J, Lutton D, Lewis SE. Sperm DNA damage has a negative association with live-birth rates after IVF. Reprod Biomed Online. 2013; 26:68-78.

Smith R, Kaune H, Parodi D, Madariaga M, Morales I, Ríos $\mathrm{R}$, Castro A. [Extent of sperm DNA damage in spermatozoa from men examined for infertility. Relationship with oxidative stress]. Rev Med Chil. 2007; 135: 279-86.

Souza AP, Santos JR, Santos TA. [The Significance of Sperm Chromatin Integrity in Male Infertility] Acta Urol 2010; 27:37-47.

Spano M, Bonde JP, Hjollund HI, Kolstad HA, Cordelli E, Leter G. Sperm chromatin damage impairs human fertility. The Danish First Pregnancy Planner Study Team. Fertil Steril 2000; 73: 43-50.

Tavalee M, Razavi S, Nasr-Esfahani MH. Influence of sperm chromatin anomalies on assisted reproductive technology outcome. Fertil Steril 2009; 91: 1119-26.

Tesarik J, Greco E, Mendza C. Late, but no early, paternal effect on human embryo development is related to sperm DNA fragmentation. Hum Reprod 2004; 19: 611-5.

Veeck L. Preembryo grading. In: Veek LL, ed. Atlas of human oocyte and early conceptus. Vol. 2. Baltimore: Williams \& Wilkins, 1991: 121-44.

Zini A, Sigman M. Are tests of sperm DNA damage clinically useful? Pros and cons. J Androl 2009; 219-29. 